\title{
Abernethy Malformation and the Spectrum of Imaging Findings: A Case Report
}

\author{
Gangadhar K, Nair SV, Sunnapwar A, Chintapalli KN
}

Department of Body Imaging and Intervention, UTHSCSA, San Antonio, USA

\begin{abstract}
Congenital extrahepatic porto systemic shunt (CEPS) is a rare condition in which the porto mesenteric blood drains into a systemic vein, bypassing the liver through a complete or partial shunt. It is commonly associated with multiple congenital anomalies. A diagnosis of Abernethy malformation is made by non-invasive cross-sectional imaging techniques such as Doppler ultrasound, CT or MRI, which show the shunt and intrahepatic portal vein branches. We report a case presenting with psychological issues, systemic hypertension, upper extremity deformities, liver lesions and portal hypertension. Usually the patients are diagnosed in childhood, in our case the patient is a middle aged woman. This case report shows the importance of imaging in diagnosis of this condition and presentation of the patient with portal hypertension which is rare.
\end{abstract}

Keywords: Abernethy malformation, congenital extrahepatic porto systemic shunt, Congenital portosystemic shunts, Nodular regenerative hyperplasia, Skeletal anomalies.

\section{Case Report}

36 year old female with history of hypertension, depression, congenital anomaly with upper extremity deformities (short forearms, 4 digits) presented with 2 day history of left facial swelling after a 2 day course of Clarithromycin for sinusitis. No lips or tongue swelling was reported. Her mother also noticed yellow sclera. Patient denied history of recent fever, chills, SOB, nausea, vomiting, diarrhea, hemoptysis, hematochezia, or melena. Patient denied

Corrospondence to: Kiran Gangadhar, Department of Body imaging and intervention, UTHSCSA, San Antonio, USA. Email: kirang.585@googlemail.com history of previous transfusions, tattoos or recreation drug use and gastrointestinal bleeding. Her menstruation was regular. Physical examination revealed left facial swelling, icterus and bilateral upper extremity deformities. Laboratory evaluation revealed raised total bilirubin $7.8 \mathrm{mg} / \mathrm{dl}$ with direct bilirubin of $3.0 \mathrm{mg} / \mathrm{dl}$ and mildly raised aspartate transaminase (AST) $55 \mathrm{IU} / \mathrm{L}$, normal alanine transaminase (ALT) 14 IU/L, raised alkaline phosphatase 127 ; raised INR 1.5; reduced platelets 12500 . Other findings of increased MCV, MCHC and anisocytosis were also seen. Admitted to medicine for further evaluation with concern for possible hemolytic anemia (elevated LDH, low haptoglobin). CT abdomen revealed features of portal hypertension including 
splenomegaly and perisplenic collaterals, multiple liver masses and direct drainage of portal vein into the inferior vena cava with absence of intrahepatic portal branches. Further evaluation with MR liver revealed T1-hypointense/T2-hyperintense heterogenous enhancing diffusion non restricting masses with similar vascular finding described in CT. Histopathological evaluation with Ultrasound guided biopsy of liver masses was done and the diagnosis of nodular regenerative hyperplasia was made. Further work up with plain radiograph revealed bilateral radii aplasia, absence of second metacarpal on right and first metacarpal on left and absence of carpal bones on radial side. All the imaging findings lead to the diagnosis of Abernethy malformation with skeletal anomalies. Chest CT did not reveal any cardiac anomalies. The patient was treated for depression and systemic hypertension. Patient was referred to gastrointestinal surgery for further evaluation.

\section{Discussion}

Congenital portosystemic shunts (CPSS) cause portal blood derived from the gastrointestinal tract and other organs in the splanchnic drainage area to flow directly into the systemic circulation. In 1997, Howard and Davenport applied the Abernethy eponym to portocaval shunts and, again, recognized the type I and type II variants. As a consequence portal blood bypasses the liver and is not subjected to hepatic metabolism. ${ }^{1}$ Congenital absence of the portal vein with an end-to-side portocaval shunt was first described by John Abernethy in $1793 .^{2}$ Many variants of congenital portosystemic shunts (CPSS) have been subsequently described, and in 1994, Morgan

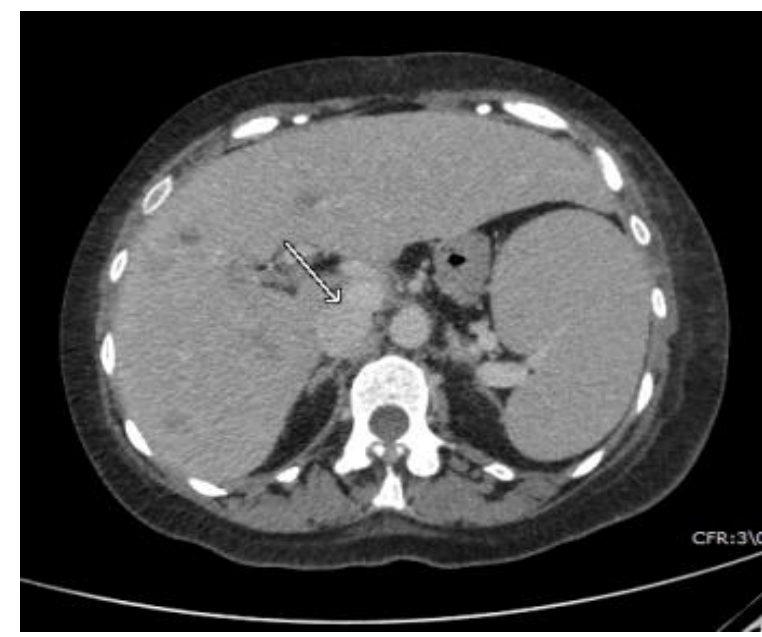

Fig. 1: Axial CECT of abdomen showing direct drainage of portal confluence into the IVC (arrow). Multiple foci of hepatic heterogeneities with splenomegaly are also noted.

and Superina introduced a classification system based on whether the portal vein, often hypoplastic, was present and whether the liver was perfused with blood from the mesenteric venous system. In a type I shunt, there is a complete end-to-side portocaval fistula with no discernable portal flow to the liver. Type II shunts occur as a side-to-side portocaval fistula or as any number of other PSS including gastrorenal, splenorenal, and portorenal shunts. The key feature of type II shunts is preservation of at least some hepatic portal flow. ${ }^{3}$ Many anatomic variations of these shunts have been recently detailed. $^{4}$

The development of the PV system is extremely complex and occurs between the $4^{\text {th }}$ and $10^{\text {th }}$ weeks of embryonic life. The PV is formed from the vitelline veins, a pair of vessels located on the anterior surface of the yolk sac. At the septum transversum (primitive liver), they are broken up into the sinusoids. Toward the end of the 4th week, three cross-communications are formed around the developing duodenum to connect 
the two vitelline veins. Selective involution of these communications generates the fully developed PV. 5

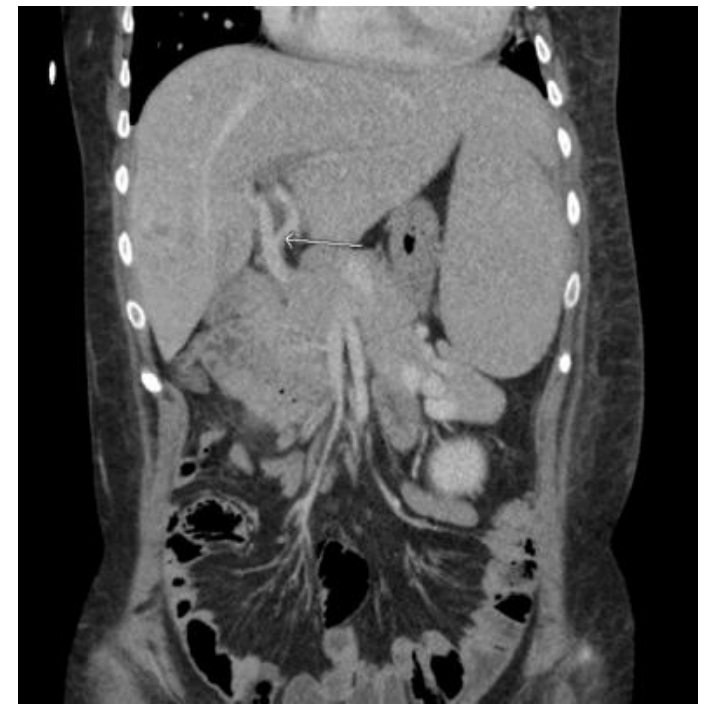

Fig.2: Coronal CECT of abdomen showing grossly dilated hepatic arteries (arrow) with absent portal vein and its intrahepatic branches. Multiple hepatic heterogeneities with splenomegaly are also noted.

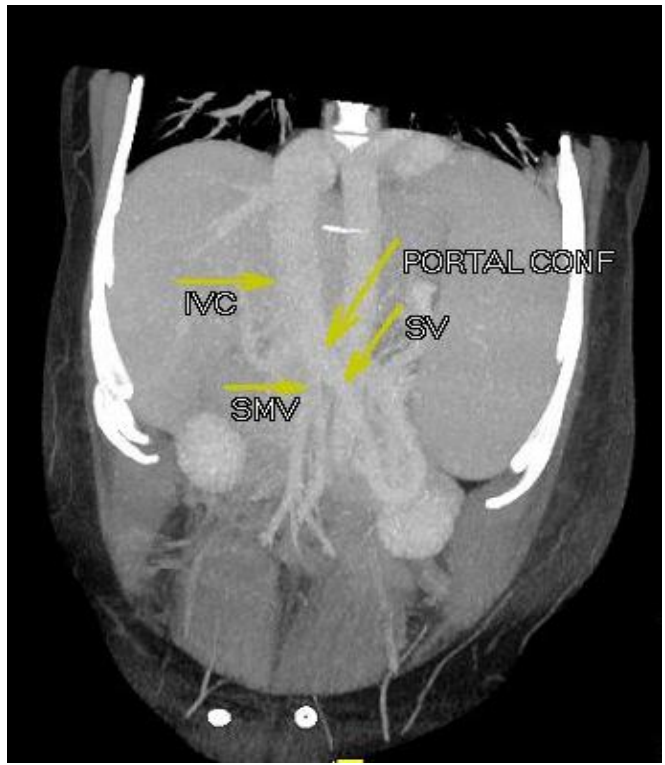

Fig. 3: Coronal Maximum intensity projection CECT abdomen showing grossly dilated hepatic arteries (arrow) without portal vein and its intrahepatic branches.
The pathogenesis of type 1 CEPSs has been attributed to excessive involution of the periduodenal vitelline venous loop or to total failure of the vitelline veins to establish the critical anastomoses with the hepatic sinusoids or umbilical veins. ${ }^{6}$ The resultant shunt may be due to persistence of the right vitelline vein (shunts draining into the retrohepatic IVC) or left vitelline vein (shunts draining into the IVC or right atrium above the level of the hepatic vein confluence) The embryologic development of the IVC is also complex. It develops from the subcardinal veins, close to the PV. The vitelline and subcardinal veins have anastomotic channels between them in the early embryonic stages; the persistence of these subcardinohepatic anastomoses has been proposed to cause type 2 CEPS. Other authors have suggested that some type 2 shunts probably arise from persistence of the right vitelline vein. ${ }^{7}$

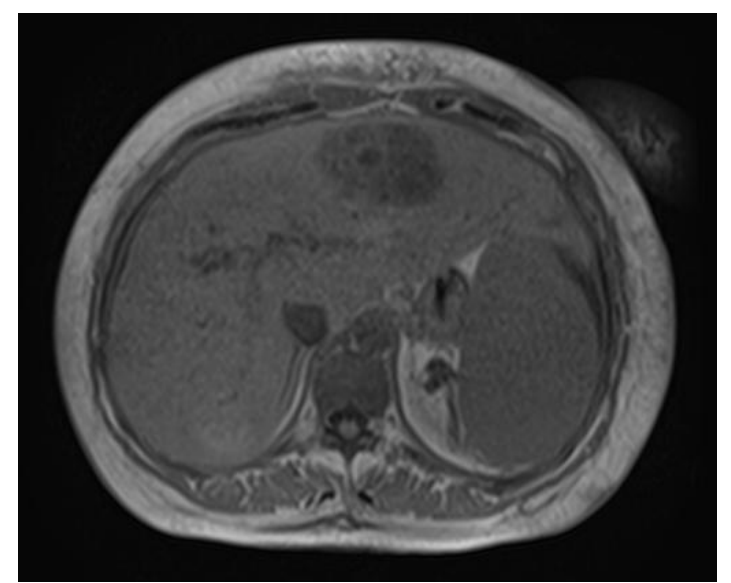

Fig. 4: Axial T1 Weighted non contrast THRIVE MR imaging of the abdomen showing hypointense heterogeneous mass lesions within the liver. Splenomegaly is also seen.

Cases of CEPS have a variable presentation. In countries having neonatal screening programmes, some cases can be diagnosed by neonatal screening tests due to presence 
of galactosemia. $^{8}$ Symptoms of secondary complications like hypoglycemia, hyperammonemia, encephalopathy and cardiac failure can be transient and can resolve spontaneously. Subclinical course is more common and some patients might not have any symptoms throughout life. ${ }^{9,11}$ Later, other lesions like nodular regenerative hyperplasia, partial nodular transformation, hepatoblastoma, hepatocellular carcinoma and adenoma can develop. ${ }^{10}$

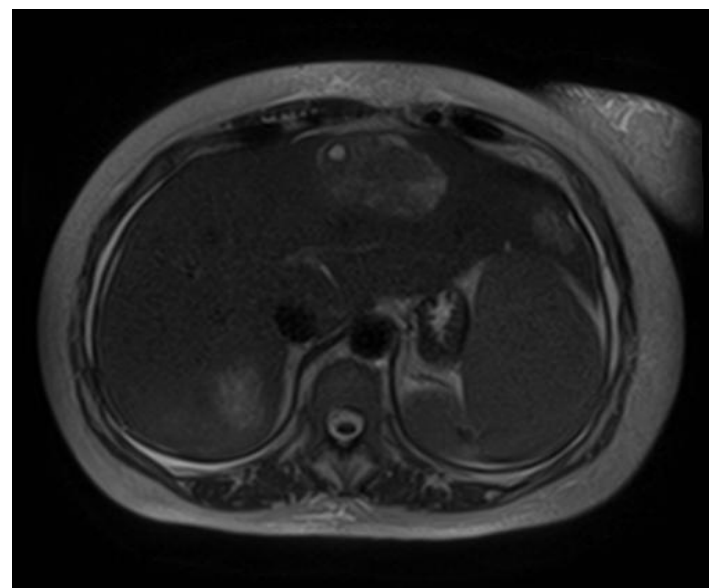

Fig. 5: Axial T2 Weighted MR imaging of the abdomen showing hyperintense heterogeneous mass lesions within the liver. Splenomegaly is also seen.

It has been proposed that the diversion of hepatotropic substances in the splanchnic venous blood, such as insulin and glucagon, away from the liver results in alterations of development, function, and regenerative capacity of the liver. This diversion, along with increased arterial hepatic flow, may contribute to the development of hepatic neoplasms. CEPS is frequently associated with other congenital anomalies, including congenital heart defects, situs ambiguous and polysplenia, malrotation, duodenal atresia, annular pancreas, skeletal anomalies, and mal-formations of the genitourinary system. They are consistently more common in type 1 CEPS, with the exception of renal tract anomalies, which are evenly represented. Many patients are diagnosed due to associated defects like heart disease, which are present in up to $60 \%$ of the patients. ${ }^{12}$

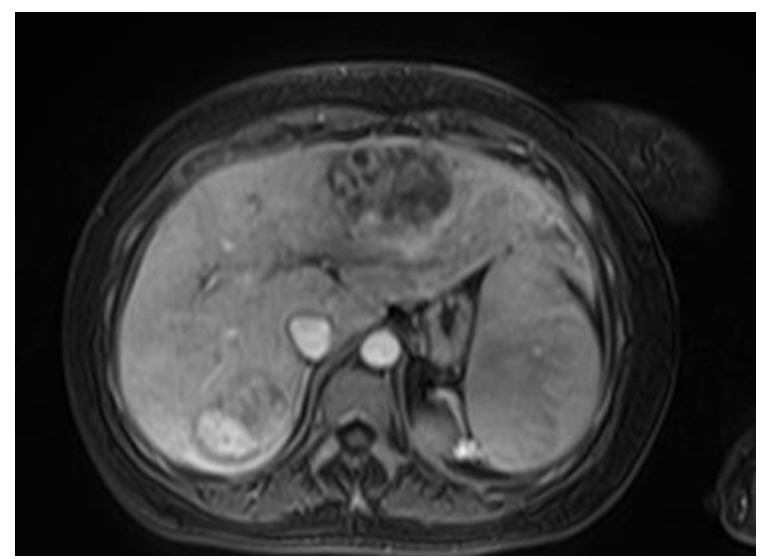

Fig. 6: Axial T1 Weighted post contrast THRIVE MR imaging of the abdomen showing heterogeneously enhancing mass lesions within the liver. Splenomegaly is also seen.

Doppler ultrasonography is the key imaging modality for the diagnosis, monitoring during the therapeutic procedure, and followup of CPSS Shunts joining the main portal vein to the inferior vena cava, end-to-side or side-to-side, may be more difficult to assess, as in most of the cases there is decreased liver size, sometimes with dysmorphy. The main finding is the nonvisibility of the intrahepatic portal branches that often appear as hyperechoic bands surrounded by hypoechoic stripes, thus giving the portal space a layered appearance. ${ }^{7}$

Multidetector computed tomography (CT) with contrast injection is the next imaging modality, to further document the anatomy and location of the shunt. Together with maximum intensity projection and multiplanar reconstruction, it provides all the necessary information about the course of the shunt, its size, and orientation; it helps to 
define the best therapeutic option and access route for radiologists or surgeons. It is also useful for the detection of complicating liver tumors. Magnetic resonance imaging (MRI) of the abdomen can also visualize the shunt, but is often less informative in small children than multidetector $\mathrm{CT}$ because of motion artifacts. However, its main indication is the evaluation of associated liver tumors, which present with variable signal intensity on T1and T2-weighted images and variable contrast enhancement. Characterization of these lesions is difficult and biopsy is needed for diagnosis. ${ }^{13}$

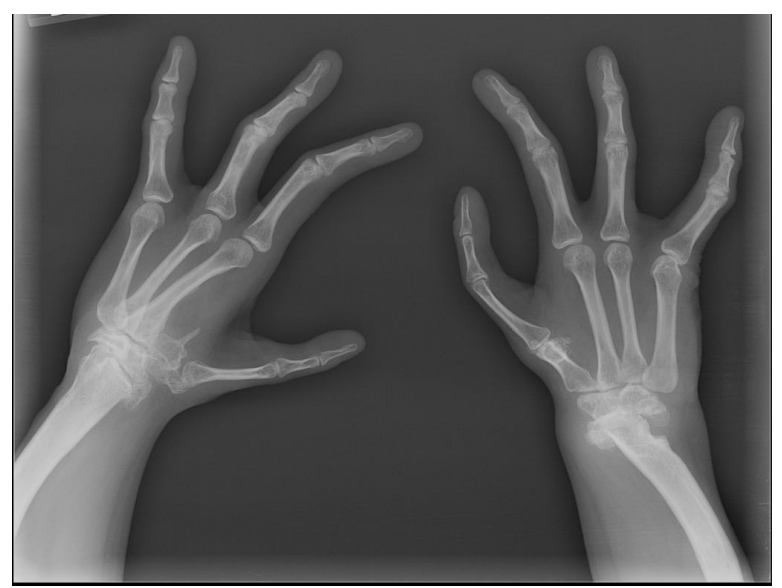

Fig. 7: Plain radiograph of the bilateral distal forearm, wrist and hand showing absence of radius, a few carpal bones, hypoplasia of the second digit on left side and aplasia of second digit on right side, and bowing of the ulna bilaterally.

Once diagnosis of CEPS is made, imaging work up for associated anomalies could be carried out. The best management approach is surgical. End-to-side shunts between the main portal vein and the inferior vena cava usually require a two-step surgical procedure to avoid acute severe portal hypertension. The first step, consisting of banding of the shunt, allows the intrahepatic portal branches to progressively develop through a cavernomatous hepatopetal network.
Complete closure a few months later can then be performed without significant portal hypertension. In complex forms of shunt, successive radiologic and surgical procedures are required to tailor the best therapeutic option for the closure of single or multiple shunts and finally to avoid liver transplantation. ${ }^{14}$

Our patient had Type $\mathrm{Ib}$ Abernathy malformation wherein a short common trunk formed by superior mesenteric and splenic vein drains directly into IVC without portal venous branches. The hepatic artery was dilated and was the sole source of vascular supply to the liver. Subclinical course is more common and some patients might not have any symptoms throughout life. ${ }^{15}$

\section{References}

1. Murray CP, Yoo SJ, Babyn PS: Congenital extrahepatic porto-systemic shunts. Pediatr Radiol 2003;33(9):614620.

2. Abernethy J. Account of two instances of uncommon formation in the viscera of the human body. Phil Trans R Soc 1793;83: 59-66.

3. Morgan G, Superina R. Congenital absence of the portal vein: two cases and a proposed classification system for portosystemic vascular anomalies. J Pediatr Surg 1994;29:1239-41.

4. Timothy B, Lautz, Tantemsapya N, Rowell E, Riccardo A. Management and classification of type II congenital portosystemic shunts. Journal of Pediatric Surgery2011;46:308-314. 
5. Howard ER, Davenport M. Congenital extrahepatic portocaval shunts: the Abernethy malformation. J Pediatr Surg 1997;32(3):494-497.

6. Altavilla G, Cusatelli P. Ultrastructural analysis of the liver with portal vein agenesis: a case report. Ultrastruct Pathol 1998;22(6):477-483.

7. Gamarra EA, Parrón M, Pérez A, Prieto C, Hierro L, Santamaría ML. Clinical and Radiologic Manifestations of Congenital Extrahepatic Portosystemic Shunts: A Comprehensive Review. RadioGraphics 2011;31:707-722.

8. Gitzelmann R, Forster I, Willi UV. Hypergalactosaemia in a newborn: selflimiting intrahepatic portosystemic venous shunt. Eur J Pediatr1997; 156(9):719-722.

9. Kim MJ, Ko JS, Seo JK, Yang HR, Chang JY, Kim GB, et al. Clinical features of congenital porto-systemic shunt in children. Eur J Pediatr; 2011.

10. Pathak A, Agarwal N, Mandliya J, Gehlot P, Dhaneria M. Abernethy malformation: a case report. BMC Pediatrics 2012;12:57.
11. Ikeda S, Sera Y, Ohshiro H, Uchino S, Uchino T, Endo F. Surgical indications for patients with hyperammonemia. J Pediatr Surg 1999;34:1012-5.

12. Raskin NH, Price JB, Fishman RA. Portal-systemic encephalopathy due to congenital intrahepatic shunts. N Engl J Med 1964;270:225-229.

13. Bernard O, Abella FS, Branchereau, Pariente D, Gauthier F, Jacquemin E. Congenital Portosystemic Shunts in Children: Recognition, Evaluation, and Management. Semin Liver Dis 2012; 32:273-287.

14. Ding XY, Chen F, Zhao XX, Wu H, Chen SP, Qin YW. A Rare Cause of Cyanosis: Hepatopulmonary Syndrome Caused by Congenital Extrahepatic Portosystemic Shunt. Hindawi Publishing Corporation Case Reports in Vascular Medicine Volume 2011.

15. Sanada Y, Urahashi T, Ihara Y, Wakiya T, Okada N, Yamada N, et al: The role of operative intervention in management of congenital extra-hepatic porto-systemic shunt. Surgery 2011,15(3):404-411. 